\title{
A Data Science Approach to Flagging Non-Retention in Engineering Enrollment Data
}

\section{Mariem Boujelbene, University of Louisville}

Mariem Boujelbene holds B.S. and M.S. degrees in Computer Science and Computer Engineering. She is currently pursuing a doctoral degree in Computer Science and Engineering and is a researcher at the Knowledge Discovery \& Web Mining Lab, Department of Computer Science and Computer Engineering, University of Louisville. Her research interests are clustering, explainability and interpretability, and recommender systems.

\section{Mr. Khalil Damak, University of Louisville}

Khalil Damak is a Ph.D. student in Computer Science and Engineering at the University of Louisville where he is also a researcher at the Knowledge Discovery and Web Mining Lab. He holds an M.S. degree in Computer Science and Engineering from University of Louisville and Bachelors from Tunisia Polytechnic School. His research mainly focuses on explainability in machine learning with applications to song explainable recommendation and sequence classification. His other research experience includes data science and machine learning on education data for student retention analysis and on autopsy and pediatric forensic reports for child abuse detection.

\section{Asuman Cagla Acun Sener, University of Louisville}

Asuman Cagla Acun Sener holds B.S. and M.S. degrees in Computer Science and Computer Engineering. She is currently pursuing a doctoral degree in Computer Science at Knowledge Discovery and Web Mining Lab, Department of Computer Science and Computer Engineering, University of Louisville. She is also working as a graduate assistant. Her research interests are educational data mining, visualization, predictive modeling, and explainability.

\section{Dr. Jeffrey Lloyd Hieb, University of Louisville}

Jeffrey L. Hieb is an Associate Professor in the Department of Engineering Fundamentals at the University of Louisville. He graduated from Furman University in 1992 with degrees in Computer Science and Philosophy. After 10 years working in industry, he returned to school, completing his Ph.D. in Computer Science Engineering at the University of Louisville's Speed School of Engineering in 2008. Since completing his degree, he has been teaching engineering mathematics courses and continuing his dissertation research in cyber security for industrial control systems. In his teaching, Dr. Hieb focuses on innovative and effective use of tablets, digital ink, and other technology and is currently investigating the use of the flipped classroom model and collaborative learning. His research in cyber security for industrial control systems is focused on high assurance field devices using microkernel architectures.

\section{Dr. Campbell R Bego, University of Louisville}

An instructor and postdoctoral researcher in engineering education, Campbell R. Bego, $\mathrm{PhD}, \mathrm{PE}$, is interested in improving STEM student learning and gaining understanding of STEM-specific learning mechanisms through controlled implementations of evidence-based practices in the classroom. Dr. Bego has an undergraduate Mechanical Engineering degree from Columbia University, a Professional Engineering license in the state of NY, and a doctorate in Cognitive Science.

\section{Dr. Patricia A Ralston, University of Louisville}

Dr. Patricia A. S. Ralston is Professor and Chair of the Department of Engineering Fundamentals at the University of Louisville. She received her B.S., MEng, and PhD degrees in chemical engineering from the University of Louisville. Dr. Ralston teaches undergraduate engineering mathematics and is currently involved in educational research on the effective use of technology in engineering education, the incorporation of critical thinking in undergraduate engineering education, and retention of engineering students. 
She leads a research group whose goal is to foster active interdisciplinary research which investigates learning and motivation and whose findings will inform the development of evidence-based interventions to promote retention and student success in engineering. Her fields of technical expertise include process modeling, simulation, and process control.

\section{Prof. Olfa Nasraoui , University of Louisville}

Olfa Nasraoui is Professor of Computer Engineering and Computer Science, Endowed Chair of e-commerce, and the founding director of the Knowledge Discovery and Web Mining Lab at the University of Louisville. She received her Ph.D. in Computer Engineering and Computer Science from the University of MissouriColumbia in 1999. From 2000 to 2004, she was an Assistant Professor at the University of Memphis. Her research activities include Data Mining/ Machine Learning, Web Mining, Information Retrieval and Personalization, in particular in problems involving large multiple domain, high dimensional data, such as text, transactions, and social network data. She is the recipient of the National Science Foundation CAREER Award, and the winner of two Best Paper Awards, a Best Paper Award in theoretical developments in computational intelligence at the Artificial Neural Networks In Engineering conference (ANNIE 2001) and a Best Paper Award at the Knowledge Discovery and Information Retrieval conference in Seville, Spain (KDIR 2018). She has more than 200 refereed publications, including over 50 journal papers and book chapters and 12 edited volumes. Her research has been funded notably by NSF and NASA. She serves since 2019 as the PI of University of Louisville's NSF funded ATHENA ADVANCE initiative. Between 2004 and 2008, she has co-organized the yearly WebKDD workshops on User Profiling and Web Usage Mining at the ACM KDD conference. She has served on the program committee member, track chair, or senior program committee of several Data mining, Big Data, and Artificial Intelligence conferences, including ACM KDD, WWW, RecSys, IEEE Big Data, ICDM, SDM, AAAI, etc. In summer 2015, she served as Technical Mentor/Project Lead at the Data Science for Social Good Fellowship, in the Center for Data Science and Public Policy at the University of Chicago. She is a member of ACM, ACM SigKDD, senior member of IEEE and IEEE-WIE. She is also on the leadership team of the Kentucky Girls STEM collaborative network. 


\title{
A Data Science Approach to Flagging Non-Retention in Engineering Enrollment Data
}

\begin{abstract}
This research paper discusses a new, data-driven metric for measuring retention. First and second year retention and retention rates are now well established as metrics in the engineering education research landscape, with many research studies exploring the impact of individual performance, noncognitive, and preparation characteristics on retention in engineering. Researchers at the University of Louisville, a large Research Institution in the Midwest, have compiled survey results and enrollment data for students in the engineering college since 2010, with the intention of conducting retrospective studies of engineering retention using this data. Using "degree earned in six years or less" to label students as retained makes over half the dataset unusable. First and second year retention are options, but these can have both false positives and false negatives. Using a data science pipeline, we analyzed the number of consecutive non-enrolled terms, referred to as enrollment gaps, and found that the best short-term criteria is "three consecutive semesters not enrolled in engineering." With this criterion, we can reliably label a given student as not-retained. The proposed retention threshold approach has the following advantages: It does not rely on the requirement of earning a degree in engineering and could be applied across a variety of fields of study, it is not based on enrollment at a fixed point in time, and it can be used as the data set continues to grow. Most importantly, while other common heuristics use grades, success in certain consecutive courses, or even demographics; our method only uses enrollment (and hence enrollment gap) data. This is a significant advantage given that the enrollment data is always available; whereas other commonly used feature heuristics for retention determination are not always available or may only apply to subsets of students.
\end{abstract}

\section{Introduction}

National reports such Rising above the gathering storm [1] have focused national attention on the fact that, historically, around half of engineering students leave engineering before completing a degree [2], [3]. Those same reports have also identified a future need for more engineers and greater diversity in engineering, a need that would be difficult to meet if a large percentage of engineering students continue to leave engineering before completing a degree. As colleges and universities respond to this national discussion there is more and more attention on retention rates in engineering. First and second year retention and retention rates are now well established as metrics in the engineering education research landscape [4], [5]. 
First year retention typically indicates that a student who entered the engineering program as a freshman in the fall of their first semester in college is still enrolled in engineering the following fall semester (with the retention rate being the percentage for an entire entering class). Similarly for second year retention. Retention in this case is synonymous with enrollment. This retention metric has been successful in identifying many performance and noncognitive factors that are significant within the first and second year of engineering school. Numerous studies have examined how a variety of performance indicators such as success in math and physics impact first and second year retention [5], [6], [7], whereas other studies have looked at other characteristics of engineering students such as belonging and motivation [8], [9], [10], [11].

Although effective for some analyses, the cross-sectional nature of the first-year retention metric results in both false positives and false negatives with respect to long-term retention and graduation. Students who are enrolled in the first semester of their second year can leave prior to graduation, and students who are not enrolled can re-enroll and complete a degree. Longitudinal techniques for measuring retention have also been investigated, such as pathway analysis [12, 13], hierarchical discrete-time event history analysis [14], domains of progression [15], support vector machines [16], Markov chains [17, 18], and critical course completion [19]. These fully longitudinal analyses require at least six years of data to begin to determine policy effectiveness and course modifications.

In this paper, we investigate a data-science-driven approach to more accurately measuring retention in the short-term using longitudinal enrollment. Our objective is not to identify the factors for retention, but rather to extract a simple data-driven rule to determine whether historic data from a student should be considered as a retention case. As we will show, the retention rule does not depend on any specific course, semester, major, or demographics, but rather on the sequential pattern of enrollment gaps throughout several semesters.

At the University of Louisville, survey data on incoming engineering students has been collected since 2010, and used to support a variety of prospective studies using survey responses. Many of those research studies have paired survey responses with enrollment data, to determine first and second year retention of students, using the definition of first and second year retention above. Recent IRB approval allowed broadening the institutional data to include additional demographic items as well as selected performance data (grades in specific courses). Though the size of the data set is not nearly as large as the MIDFIELD data set [20], the data set now covers a period of ten years and includes survey responses from more than 4000 freshman engineering students and semester by semester enrollment, graduation and performance data for over 6000 engineering students.

Because the data set is continuing to grow (survey responses, student enrollment and performance data as well as demographic data are added each year), retrospective studies focused on retention would benefit from a consistent labeling of students as retained or not 
retained. Students who earn an engineering degree would be one such labeling; however, while some students complete a degree in four years, others complete it in six, or even seven years. If earning a degree in six years or less is used as the definition for retained, then less than half of the current data set would be usable. Retained first year and retained second year are another possible labeling. However, due to phenomena such as stopout (leaving and reentering an engineering program) [4] and the continued attrition of engineering students into the second and even third year, these labelings have both false positives and false negatives. Our study which seeks to identify, using a data science approach, a consistent way to label all students as either retained or not retained, enjoys the following advantages:

a) It does not rely on the requirement of earning a degree in engineering,

b) It is not based on enrollment at a fixed point in time, and

c) It can be used as the data set continues to grow.

Using a data science pipeline, we analyze student enrollment gaps to determine a reasonable labeling of not-retained. In the following, we start by describing our methods, then present our findings and finish with a discussion and conclusions.

\section{Methods}

\section{A Data-driven Pipeline for Retention Analysis}

The goal of this study is to define a data-driven heuristic to identify non-retention in Engineering. We looked specifically at enrollment gaps, in other words, the number of consecutive non-enrolled terms. The heuristic identified a threshold number of terms that would give the most accurate prediction of retention. Our study involves undergraduate students in Engineering.

\section{Data description}

The data used contains the enrollment and graduation status of a sample of 6,383 students from the Speed School of Engineering at University of Louisville, of whom 5,200 are undergraduates. In this study, we only focus on undergraduates, thus we filtered out the graduate students. The data includes enrollment and graduation data from Fall 2010 to Spring 2018. In every term, we have a list of enrolled students and a list of graduates. Fig. 1 shows the distribution of the number of undergraduate students per academic year and term.

Among all the undergraduate students, 1,803 (28.24\%) have graduated with at least one degree, as shown in Fig. 2 which depicts the distribution of degrees in our data. These students are considered as retained. The students that did not earn a degree are either not-retained or are still in the process of graduation. Thus, our aim is to distinguish the latter two status possibilities. 


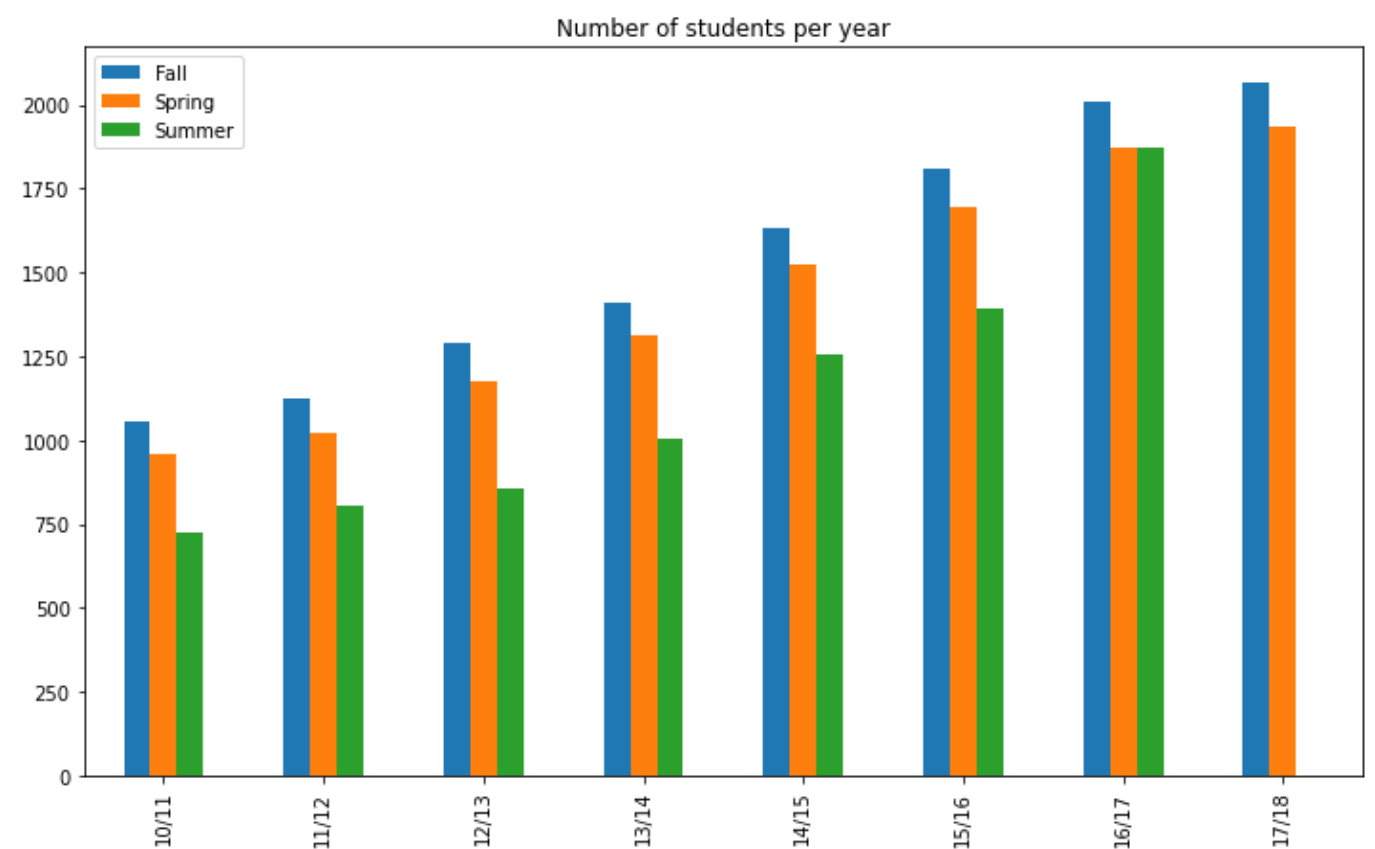

Figure 1: Distribution of the number of undergraduate students per academic year and term

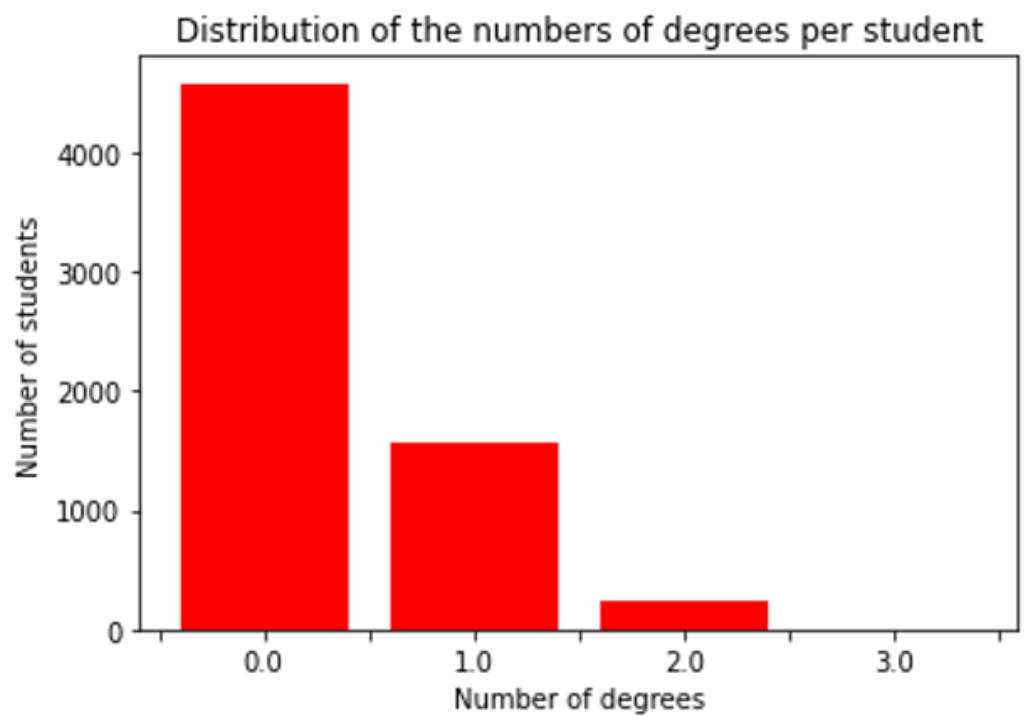

Figure 2: Distribution of number of degrees.

Table 1: Distribution of number of degrees.

\begin{tabular}{|l|l|l|l|l|}
\hline $\begin{array}{l}\text { Number of } \\
\text { degrees }\end{array}$ & 0 & 1 & 2 & 3 \\
\hline $\begin{array}{l}\text { Number of } \\
\text { students }\end{array}$ & 4580 & 1570 & 230 & 3 \\
\hline $\begin{array}{l}\% \text { of } \\
\text { students }\end{array}$ & 71.75 & 24.59 & 3.6 & 0.04 \\
\hline
\end{tabular}




\section{Data-driven Retention Threshold Determination}

We started by merging the data files of all the terms by the student ID. Then, we encoded the data to obtain sequences of consecutive events, corresponding to the consecutive enrolled-terms of the student. Likewise, we mapped to a sequence of zeroes, all the consecutive non-enrolled terms. Naturally, we did not consider the terms that preceded the initial enrollment of the student or the terms that followed their graduation. The aforementioned process is depicted in Fig. 3. Finally, we used the distribution of consecutive non-enrolled terms to define a threshold for retention determination.

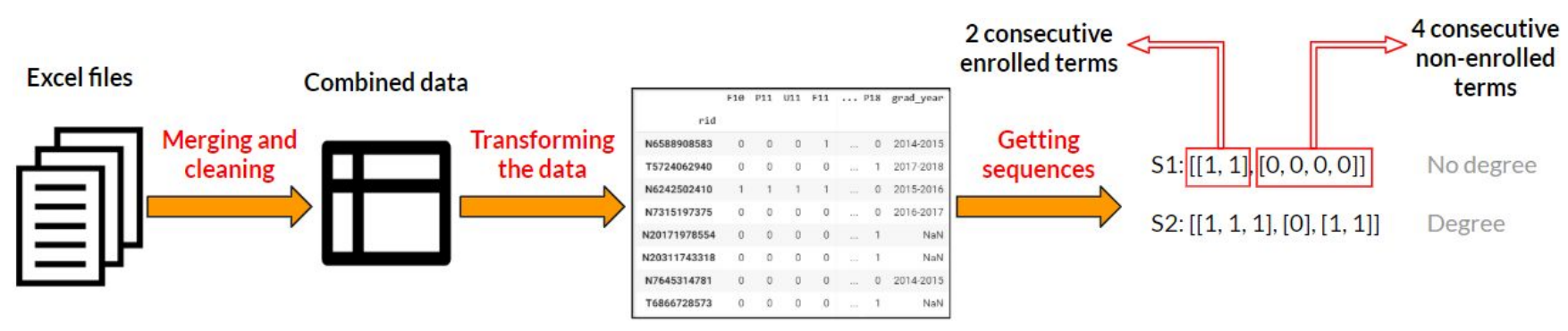

Figure 3: Data preprocessing pipeline for retention threshold determination

The aforementioned process is detailed further in the following steps:

- Encode enrolled and non-enrolled terms for every student in a binary vector, with as many elements as the total number of terms, and with each element ( 1 or 0$)$ indicating whether a student is enrolled (1) or not (0) in the corresponding term.

- Label the students as "graduated" or not.

- Convert every row into a list, whose entries consist of the count of the consecutive ones and zeroes corresponding, respectively, to enrolled and non-enrolled terms.

- Remove the zeroes preceding the appearance of the first 1 in each list. We assumed that the first 1 denotes the initial enrollment of the student.

- If the student graduated, then remove the sequence of zeroes following the last 1 . We assume that the last enrolled semester corresponds to the graduation semester.

- Count the lengths of the consecutive sequences of zeroes.

- Plot the distribution of the number of consecutive non-enrolled terms as a function of the graduation status to determine the threshold for retention.

\section{Results}

Fig. 4 shows the distribution of non-enrolled terms for graduated and non-graduated students. The intuition behind this method is to find a visually salient threshold after which the difference between the number of graduated and non-graduated students becomes relatively high. This means that after this certain number of non-enrolled terms, the likelihood of a student graduating becomes relatively low. 


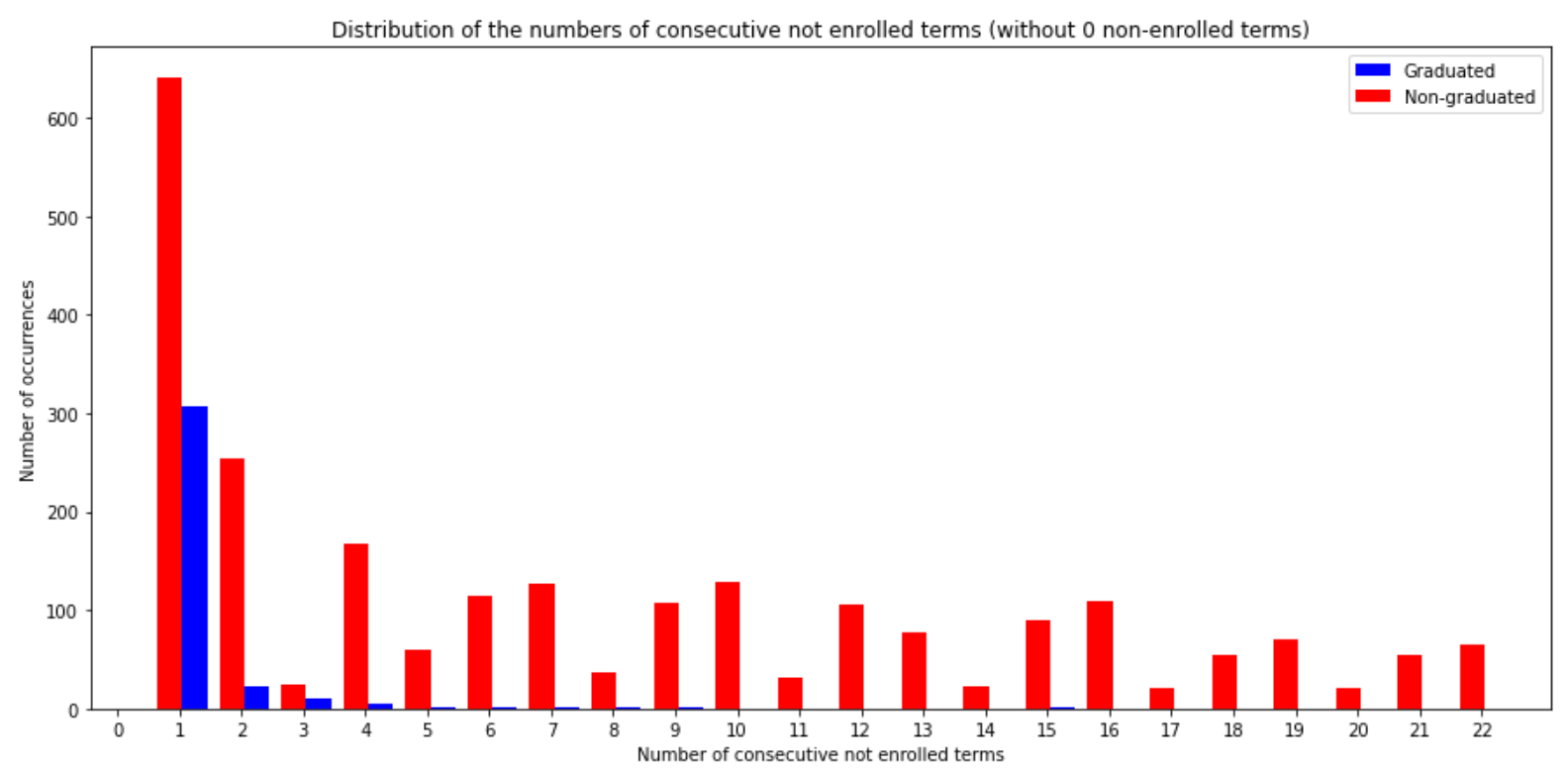

Figure 4: Distribution of the number of consecutive non-enrolled terms for graduates vs. non-graduates (we removed 0 terms for better visibility)

One way to choose the threshold for the number of consecutive non-enrolled semesters, is to compute the probability of misclassification of a student in the non-retention category given a particular retention cutoff threshold, then choose the threshold that minimizes the misclassification probability. Let $k$ be the number of consecutive non-enrolled semesters that could be used as a candidate retention cutoff threshold, let $C_{k}$ be the set of correctly classified students for threshold $k$, and let $M_{k}$ be the set of misclassified students for threshold $k . M_{k}$ is the set of graduated students for whom the number of consecutive non-enrolled semesters has exceeded the threshold $k$ at any given time. Let $T_{k}$ be the set of students for whom, given a threshold $k$, the number of consecutive non-enrolled semesters is higher than $k$. Equation 1, below, defines the probability that a new student would be misclassified if the threshold is set to $k$.

$$
P\left(s \in M_{k} \mid k\right)=\left|M_{k}\right| /\left|T_{k}\right|
$$

The probability that a student would be correctly classified as not retained can be computed using equation 2 , below:

$$
P\left(s \in C_{k} \mid k\right)=1-P\left(s \in M_{k} \mid k\right)
$$

In order to choose the threshold $k$ that minimizes the probability that a student will be misclassified (or equivalently maximizes the probability that a student will be correctly classified), we plot the probability of misclassification for all possible thresholds, as shown in Fig. 5. 


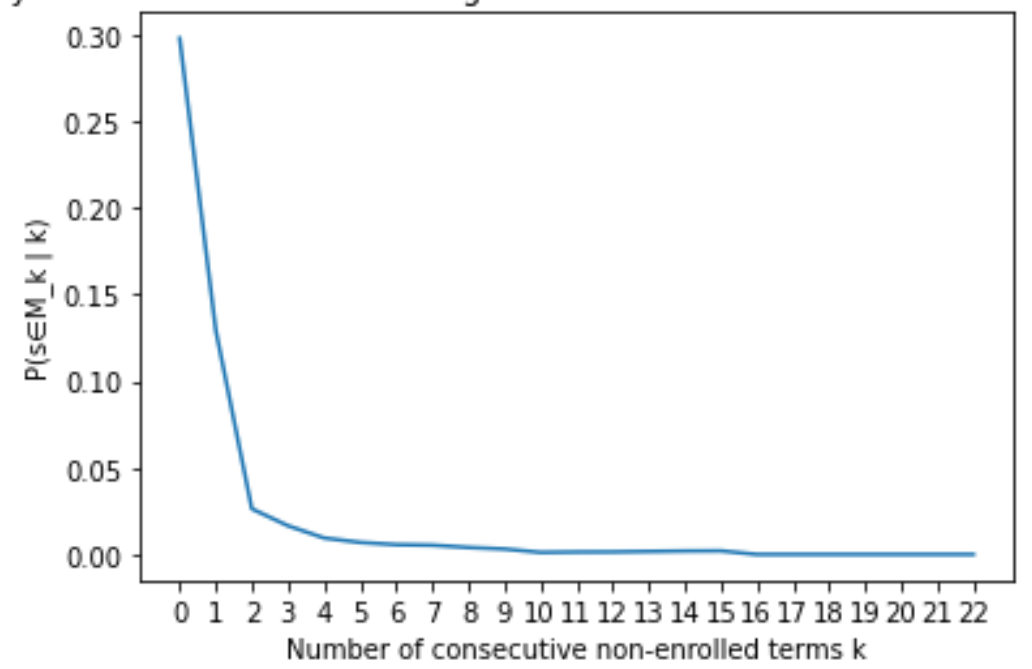

Figure 5: Probability of misclassifying a student in the non-retention category versus the number of consecutive non-enrolled terms $k$

The probability of retention misclassification in Fig. 5 decreases with the threshold of consecutive non-enrolled terms. We notice an elbow-shape pattern that represents a fast decrease, followed by a plateau where the probability slowly vanishes to zero. The elbow's location between $\mathrm{k}=2$ and 4 , suggests a threshold of 3 consecutive non-enrolled semesters, above which a student may be considered as not retained. This threshold results in a probability of error of 0.016 , meaning that if a student is not enrolled for at least three consecutive semesters, we can categorize the student as not retained, with a probability of at least $98 \%$.

\section{Discussion and Conclusion}

Without a standard way to define retention, retrospective studies would likely each develop their own definition. Using a data-driven heuristic for the definition of retention allows independent retrospective studies to maintain a consistent definition of retained, and for researchers to have a common ground in terms of retention. It also alleviates the need for researchers to define retention, something they often have not thought about in this very practical way.

Our data science approach is a novel and practical method to find the criteria to label student retention. Our proposed heuristic for retention determination mainly differs from state of the art heuristics in the time-dependent data needed to flag students who have dropped out. In fact, while other more common heuristics use less accessible data points such as grades, success in consecutive courses, or even demographics; our method only uses enrollment data. This is a significant advantage given that the enrollment data is always available, whereas other commonly used feature heuristics for retention determination are not always available 
or may only apply to subsets of students. Furthermore, our method may be extended to other fields and other education levels. In fact, the same study could be applied to graduate-level students to determine another data-driven retention threshold. For this case and other specific cases, methods based on course-success, for example, could be limited by the fact that there are no common fundamental courses that students need to take in a certain order.

In addition to offering a flexible mechanism to determine retention, the proposed approach allows all labels to be updated when adding new data. Although labels might change with the addition of more data, the labelling process remains consistent and the data-driven approach allows revising the retention cutoff threshold dynamically as the data gets updated.

One limitation of the proposed approach comes from its heuristic nature and the difficulty to verify the threshold due to the absence of a ground truth retention flag.

We conclude that for our dataset at the University of Louisville, a good short-term metric for non-retention is a 3 -semester enrollment gap. This metric avoids the false-positives and false negatives of a single cross-sectional measure of first-year retention, and does not require six years of longitudinal data. We recommend that other universities apply the proposed data-driven approach for determining retention metrics for their unique programs. 


\section{References}

[1] Augustine, N. R. (2005). Rising above the gathering storm: Energizing and employing America for a brighter economic future. Retrieved March, 19, 2008.

[2] Chen, X. (2013). STEM Attrition: College Students' Paths into and out of STEM Fields. Statistical Analysis Report. NCES 2014-001. National Center for Education Statistics.

[3] Olson, S., \& Riordan, D. G. (2012). Engage to Excel: Producing One Million Additional College Graduates with Degrees in Science, Technology, Engineering, and Mathematics. Report to the President. Executive Office of the President.

[4] Min, Y., Zhang, G., Long, R. A., Anderson, T. J., \& Ohland, M. W. (2011). Nonparametric survival analysis of the loss rate of undergraduate engineering students. Journal of Engineering Education, 100, 349-373.

[5] Ohland, M. W., Yuhasz, A. G., \& Sill, B. L. (2004). Identifying and removing a calculus prerequisite as a bottleneck in Clemson's General Engineering Curriculum. Journal of Engineering Education, 93, 253-257.

[6] Felder, R. M., Forrest, K. D., Baker-Ward, L., Dietz, E. J., \& Mohr, P. H. (1993). A longitudinal study of engineering student performance and retention: I. Success and failure in the introductory course. Journal of Engineering Education, 82, 15-21.

[7] Hensel, R., Sigler, J., \& Lowery, A. (2008). AC 2008-2079: BREAKING THE CYCLE OF CALCULUS FAILURE: MODELS OF EARLY MATH INTERVENTION TO ENHANCE ENGINEERING RETENTION. Paper presented at the Proceedings of the 2008 American Society for Engineering Education Annual Conference \& Exposition, Pittsburgh, PA.

[8] Honken, N. B., \& Ralston, P. (2013). Freshman Engineering Retention: A Holistic Look. Journal of STEM Education: Innovations and Research, 14.

[9] Honken, N. B., \& Ralston, P. A. S. (2013). High-Achieving High School Students and Not So High-Achieving College Students A Look at Lack of Self-Control, Academic Ability, and Performance in College. Journal of Advanced Academics, 24, 108-124.

[10] Moses, L., Hall, C., Wuensch, K., De Urquidi, K., Kauffmann, P., Swart, W., Dixon, G. (2011). Are Math Readiness and Personality Predictive of First-Year Retention in Engineering? The Journal of Psychology, 145, 229-245.

[11] Weaver, J. P., DeCaro, M. S., Hieb, J. L., \& Ralston, P. A. Social Belonging and First-Year Engineering Mathematics: A Collaborative Learning Intervention. In ASEE Conferences, New Orleans, Louisiana. 2016. 
[12] R. Robinson, "Pathways to completion: Patterns of progression through a university degree," High. Educ., vol. 47, pp. 1-20, 2004, doi: 10.1023/B:HIGH.0000009803.70418.9c.

[13] R. Molontay, N. Horvath, J. Bergmann, D. L. Szekrenyes, and M. Szabo, "Characterizing curriculum prerequisite networks by a student flow approach," IEEE Trans. Learn. Technol., 2020, doi: 10.1109/tlt.2020.2981331.

[14] P. R. Bahr, "Educational attainment as process: Using hierarchical discrete-time event history analysis to model rate of progress," Res. High. Educ., vol. 50, pp. 691-714, 2009, doi: 10.1007/s11162-009-9135-x.

[15] J. Pienaar and X. Zhao, "Factors influencing student progression in built environment and engineering programs: Case of Central Queensland University," J. Prof. Issues Eng. Educ. Pract., vol. 143, no. 4, pp. 1-9, 2017, doi: 10.1061/(ASCE)EI.1943-5541.0000341.

[16] D. Ifenthaler and C. Widanapathirana, "Development and validation of a learning analytics framework: Two case studies using support vector machines," Technol. Knowl. Learn., vol. 19, pp. 221-240, 2014, doi: 10.1007/s10758-014-9226-4.

[17] A. M. Bessent and E. W. Bessent, "Determining the Comparative Efficiency of Schools through Data Envelopment Analysis," Educ. Adm. Q., vol. 16, no. 2, pp. 57-75, 1980, doi: $10.1177 / 0013161 X 8001600207$.

[18] C. Shah and G. Burke, "An undergraduate student flow model: Australian higher education,” High. Educ., vol. 37, pp. 359-375, 1999, doi: 10.1023/A:1003765222250.

[19] C. R. Bego, J. L. Hieb, and P. A. S. Ralston, "Barriers and bottlenecks in engineering mathematics: How performance throughout a math sequence affects retention and persistence to graduation," in October 2019 IEEE Frontiers in Education Conference (FIE), 2019.

[20] M. W. Ohland and R. A. Long, "The multiple-institution database for investigating engineering longitudinal development: An experiential case study of data sharing and Reuse," Adv. Eng. Educ., vol. 5, no. 2, pp. 1-25, 2016. 